\title{
Respiratory System Link Group
}

National Cancer Institute

\section{Source}

National Cancer Institute. Respiratory System Link Group. NCI Thesaurus. Code

C162037.

A sequence of characters used to link multiple respiratory system findings to a single finding. 